\title{
Research on the Influence of Culture Capital on Compensatory Consumption Based on Relative Deprivation Theory
}

\author{
Wenwen Feng, Hai Hu \\ Department of Finance and Economics, Nanjing Normal University, Nanjing, China \\ Email: 475997847@qq.com
}

How to cite this paper: Feng, W.W. and $\mathrm{Hu}, \mathrm{H}$. (2019) Research on the Influence of Culture Capital on Compensatory Consumption Based on Relative Deprivation Theory. Open Journal of Business and Management, 7, 1346-1357.

https://doi.org/10.4236/ojbm.2019.73093

Received: June 12, 2019

Accepted: July 16, 2019

Published: July 19, 2019

Copyright (c) 2019 by author(s) and Scientific Research Publishing Inc. This work is licensed under the Creative Commons Attribution International License (CC BY 4.0).

http://creativecommons.org/licenses/by/4.0/

\begin{abstract}
Compensatory consumption refers to the alternative consumption behavior adopted by individuals to make up for a certain psychological disadvantage or self-threat. It is a new topic worth exploring in the field of consumer behavior. In the past, the research focused on the mechanism of compensatory consumption from the perspective of social identity theory and power. However, few literatures explore the mechanism of compensatory consumption on the base of relative deprivation theory. Therefore, based on the relative deprivation theory, this research explores the mechanism of cultural capital's influence on compensatory consumption, and examines the moderating effects of social support. Through empirical analysis of 357 questionnaire data, the study found that: culture capital negatively affects relative deprivation and compensatory consumption; relative deprivation positively affects compensatory consumption and plays a mediating role between cultural capital and compensatory consumption; social support moderates the relationship between cultural capital and compensatory consumption by weakening individual relative deprivation.
\end{abstract}

\section{Keywords}

Cultural Capital, Compensatory Consumption, Relative Deprivation, Social Support

\section{Introduction}

The traditional western economic consumption research is usually based on the assumption of rational economic man, and pursuing utility maximization under the constraints of consumers' existing resources. However, in real life, there are various motives behind people's consumption behavior which is difficult to 
measure by rational economic standards. According to the China Luxury Report released by McKinsey \& Company [1], the consumption of luxury goods by Chinese consumers has reached 500 billion yuan, accounting for one-third of the total annual sales of luxury goods worldwide. In addition, the report also shows that among the luxury consumers in China, the number of young consumers (between 18 and 30 years old) is increasing which is a group of customers that cannot be ignored. However, at this stage, the younger generation in China is basically in the middle and low income level of the period when the economy cannot be independent or the beginning of the working life. The consumption of luxury goods is not proportional to its own economic ability. In order to satisfy their desire to purchase luxury goods, it is not uncommon to save money and borrow money. What's more, personal privacy information is used as collateral to purchase high-end goods from unscrupulous platform loans, and because of the high interest rate, personal privacy information may be leaked, misguided or even costly. Therefore, in the transition period of the current severe division of the class, it is particularly important to explore the inner mechanism of these "extravagance before wealth" phenomena for the harmonious development of Chinese society.

Many of the previous literatures mainly explain these irrational consumption behaviors from the perspective of conspicuous consumption. They believe that people tend to show their own financial resources and status through the consumption side of high-end brands, thus building an elite sense of superiority [2]. According to the flamboyant consumption theory of Veblen [3], conspicuous consumption refers to the wealth display behavior of individuals who possess sufficient material wealth, and thus cannot fully explain the phenomenon of "extravagance before wealth" phenomena. So, what are the causes of these economically disadvantaged groups blindly consuming high-end goods? Recent research has proposed a new interpretation-compensatory consumption. Compensatory consumption refers to the irrational consumption behavior of individuals based on psychological compensation motives to compensate for certain psychological defects or self-threat [4]. Psychologists believe that individual consumption decisions are not only affected by their actual needs, but also by their psychological needs. When the psychological needs of individuals with relatively low economic status (such as the need for interpersonal belonging and respect) are not met, they may hope to seek self-affirmation and compensate for the lack of value through consumer compensatory products [5].

At present, the literature on compensatory consumption is mainly concentrated in western countries, and domestic research in this field has just begun. In addition, the existing literature mainly explores the mechanism of compensatory consumption from the social factors such as status [6] and power level, but few scholars have noticed the role of deeper culture. Although status and power as a specific identity will have a relatively stable impact on consumer behavior [7], individuals not only have different status, but also differences in cultural level and consumption philosophy. These factors may affect consumers' impulse cha- 
racteristics, self-control ability and materialist values, which in turn affect their compensatory consumption propensity [8]. According to Bourdieu's cultural capital theory [9], cultural capital can not only initiate and maintain identity, mark status differences, but also give individuals extraordinary consumer performance. Therefore, cultural capital may be closely related to the compensatory consumption of individuals. This study takes this as an entry point and mainly explores the mechanism and boundary conditions of cultural capital affecting compensatory consumption. Specifically, based on the theory of relative deprivation, this study constructs the theoretical framework of "cultural capital-relative deprivation-compensatory consumption" and examines the moderating effect of social support in order to enrich the literature research on compensatory consumption as well as provide theoretical support for promoting the mental health development of young people.

\section{Theoretical Review and Research Hypothesis}

\subsection{Compensatory Consumption}

In the field of psychology, "compensation" refers to the psychological adaptation process in which an individual tries to achieve achievements and improve oneself in other fields in order to compensate for the perceived disadvantages of himself. Based on this, Gromo [10] creatively introduced compensation theory into the consumer field and developed the concept of compensatory consumption: compensatory consumption refers to the fact that consumers in relatively disadvantaged economic status can compensate for their lack of inner feelings through certain specific consumption behavior because of some psychological disadvantage or unsatisfactory inner needs. Grunert [11] extends the definition of compensatory consumption, which does not recognize that Gromo restricts the object of compensatory consumption to the economically disadvantaged groups. He believes that any consumer may exhibit compensatory consumption behavior. Moreover, she further explained the concept of compensatory consumption: when the resource that the individual really lacks is $\mathrm{x}$, but for various reasons, it cannot be directly compensated from $\mathrm{x}$, but can only turn to another unrelated resourcey. Similarly, Rucker and Galinky [4] introduced self-concept into compensatory consumption behavior based on the understanding of Belk's material possession theory, and considered that compensatory consumption refers to the behavior that consumers compensates by consuming products that can represent achievements in the threatening field when their self-concept (such as self-worth, self-esteem, etc.) is threatened. Through the above summary and analysis of scholars' research on compensatory consumption, this paper argues that the consumer behavior that occurs in order to compensate for the threat of perceived demand or self-concept is the compensatory consumption.

\subsection{Cultural Capital and Compensatory Consumption}

Bourdieu [9] proposes that cultural capital is a collection of tangible and intang- 
ible cultural assets acquired by individuals in the process of socialization which including habitus, unique tastes, skills, cultural knowledge and practice. According to the theory of cultural capital, cultural capital can not only promote individual knowledge accumulation and educational acquisition, but also help them to show extraordinary consumption culture and rational level in the consumption field [9]. As an irrational consumer behavior, compensatory consumption is often the negative response of individuals to their perceived psychological disadvantages and various threats [4]. Literature studies have shown that factors such as frustration of self-esteem, lack of power, and social exclusion can significantly promote the compensatory consumption propensity of individuals [5]. According to the theory of cultural reproduction, culture capital can improve individual non-cognitive ability (self-control, self-confidence and emotional stability, etc. ...) by a set of conversion of advantages [8]. Obviously the acquisition of these abilities also helps high culture capital individuals to resist the impulse of inferiority and compensatory consumption. In addition, irrational consumption is also affected by individual materialist values. The higher the individual's level of materialism, the more likely it is to compensate for the lack of inner value through the consumption of brand-name products. A good culture background can weaken this bad value, and thus reduce the compensatory consumption tendency. Based on the above reasoning, the following hypothesis is proposed:

H1: Culture capital negatively affects compensatory consumption.

\subsection{The Mediating Role of Relative Deprivation}

Relative deprivation refers to the anger or dissatisfaction caused by the inability of the individuals when they find that they are at a disadvantage when compared with the reference object [12]. According to the theory of material possession, people buy goods not only to obtain their functions, but more to represent self-concept and obtain social identity through consumption [13]. Song Xiaobing, Duan Caiwei and Wang Di's research [14] shows that individual consumption attitudes or preferences are often the embodiment of their cultural capital in the field of consumption. High-cultural capital individuals tend to show their knowledge and skills through taste consumption. On the contrary, the low-cultural capital individual lacks a clear understanding of self-concept and consumer taste on the one hand [15], and on the other hand, their symbolic appeal for rational representation of self-image and identity is constantly increasing. At this time, the relative weakness of cultural capital makes the individual in the long-term comparison with the reference group. As a result, their self-concept and belonging are faced with a double threat, thus creating a strong sense of relative deprivation. Consequently Hypothesis 2 is stated as follows:

$\mathrm{H} 2$ : Cultural capital negatively influences relative deprivation.

Relative deprivation theory believes that relative deprivation will induce some negative psychology and behavior of individuals [16]. Previous studies have shown 
that relative deprivation has significant predictive effects on individual emotional disorders, depression, and aggressive behavior [17]. Therefore, when an individual suffers consumer deprivation after comparing upwards, it is very likely that they will choose to obey the reference group norm and relieve their relative deprivation through compensatory consumption. Previous literature has also confirmed that relative deprivation has a significant predictive effect on materialism and conspicuous consumption [18] [19]. Based on the above reasoning, the following hypotheses are proposed:

H3: Relative deprivation positively affects compensatory consumption.

H4: Relative deprivation plays a mediating role between cultural capital and compensatory consumption.

\subsection{The Moderating Role of Social Support}

Social support refers to the overall degree of individual's feelings of caring, caring and respecting that they have gained in interpersonal communication, and is usually divided into instrumental social support and emotional social support [20]. Literature research shows that as a benign social resource, individuals have significant differences in emotions, attitudes, and behaviors under the different conditions of social support [21]. A high level of social support can effectively alleviate the anxiety caused by various stress events and help individuals maintain normal mental health [22]. Therefore, for individuals with relatively low cultural capital, when they are in a weak position in the consumer socialization process of the reference group, the full support from family and friends may weaken their inner relative deprivation and other negative emotions. On the contrary, the lack of social support and even social exclusion will make individuals feel more lonely and empty, and their heart will become more vulnerable and sensitive. These negative emotions are intertwined and may further exacerbate the pressure of relative deprivation brought by individuals with low culture capital. Based on the above reasoning, the hypothesis 5 is formulated.

H5: Social support plays a moderating role between cultural capital and relative deprivation.

Social support will not only moderate the relationship between culture capital and relative deprivation, but may also affect the indirect role of culture capital to compensatory consumption through relative deprivation. Specifically, the less social support an individual receives, the easier it is to generate negative feelings such as relative deprivation in the comparison with the cultural capital of the reference group, which often leads people to prefer to seek more psychological compensation through consumption activities [23]. Therefore, the relative deprivation of the individual mediates the influence of culture capital on compensatory consumption, but the level of social support felt by the individual will affect the strength of the intermediary effect. Based on the aforementioned reasoning, hypothesis 6 is proposed.

H6: Social support moderates the indirect effects of cultural capital on com- 
pensatory consumption.

In summary, the theoretical model framework of this study is shown in Figure 1.

\section{Research Design}

\subsection{Research Sample}

In this study, questionnaires were used to collect data through online and offline channels. The questionnaire included demographic factors such as gender and age as well as key variables involved in the study. A total of 464 questionnaires were distributed, 184 for paper questionnaires and 247 for network questionnaires, totaling 431. After eliminating the invalid questionnaire, 357 valid samples were obtained, and the effective responserate was $76.94 \%$. In addition, a statistical description of all valid samples found that male samples accounted for $36.1 \%$, females accounted for 63.9\%; most of the participants were between 18 and 40 years old, and with basically undergraduate higher education; furthermore, their monthly disposable (main income) is between 1000 and 3000, and $41.5 \%$ of the participants are mainly based on students, teachers and enterprises in their occupational distribution.

\subsection{Measurement Tools}

The questionnaires used in this study are mainly the measurement tools developed and published by authoritative journals at home and abroad, and the validity of the questionnaire content is verified by the proposed two-way translation program by Brislin (1980). In addition, the analysis of these data is done by Mplus7.0 and the Statistical Product and Service Solutions, SPSSversion22.0, an IBM product since 2009 [24]. Specifically, the measurement of cultural capital mainly refers to the scales used in the research of Song Xiaobing, Duan Caiwei and Wang Di [14], which including the three items, and the Cronbach's alpha is 0.837 ; the relative deprivation is measured according to the scale compiled by Ma Wei [12], (2012) including four items, and the Cronbach's alpha is 0.803 ; the measurement of compensatory consumption is based on the research of Jin Xiaotong, Zhao Sun and Cui Hongjing [25], which includes three items, and the Cronbach's alpha is 0.811 . The variables involved in the above studies were all scored using the Likert 5 point scale, where 1 represents "completely non-conformance" and 5 represents "completely consistent". The measurement of social support refers to the research of Xiao Shuiyuan [20], which includes 10 items, scored by 4 points, and the Cronbach's alpha is 0.919. It can

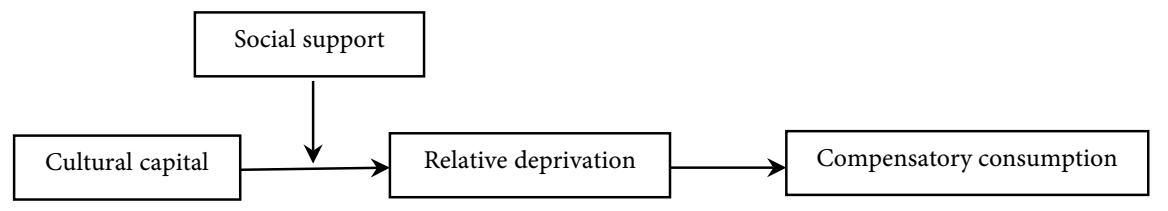

Figure 1. Conceptual framework model. 
be seen that the reliability coefficients of each scale are greater than 0.7 , indicating that the scale selected in this study has good reliability. According to Hejase and Hejase [24], Cronbach's alpha values between 0.80 and above are very good to excellent internal reliability measures. In addition, in order to avoid the impact of systematic errors caused by other variables on compensatory consumption, this paper draws on the previous literature, taking the individual's gender, age, occupation, education level, and monthly disposable income as control variables.

\section{Hypotheses Testing and Research Results}

\subsection{Common Method Variance Test}

Since all the data involved in this study were obtained by the observation object at the same time based on the self-assessment of the questionnaire items, the problem of common method variance (CMV) may occur. Therefore, the Harman single factor analysis method was used for the test. Specifically, with SPSS22.0 software, exploratory factor analysis is performed on all items of the sample, and the unrotated factor is extracted according to the principle that the feature value is greater than 1 . The analysis results show that among the four common factors formed, the variance contribution rate of the first principal component is $34.35 \%$, which is lower than half of the total variation, indicating that there is no serious common method variance in this study.

\subsection{Confirmatory Factor Analysis and Validity Test}

This article performs a confirmatory factor analysis of all variables using Mplus software. The results show that the normalized factor loading (STD.) of all variables is greater than 0.6 , the composition reliability (CR) is greater than 0.7 , and the average extraction amount (AVE) is greater than 0.5 , indicating that the model has good convergence validity. In addition, the AVE square root of each variable shown in Table 1 is also greater than the correlation coefficient between it and other latent variables, indicating that the variables have higher discriminant validity. Furthermore, the basic model's fitness indicators also reach the relevant standards $\left(\chi^{2} / \mathrm{df}=1.322, \mathrm{CFI}=0.984, \mathrm{TLI}=0.982, \mathrm{RMSEA}=0.030\right.$, SRMR $=0.036$ ), which can be considered as the structural effect of the basic model is relatively high.

Table 1. Descriptive statistics.

\begin{tabular}{lllllll}
\hline Variable & Mean & \multicolumn{1}{l}{ Standard deviation 1} & 2 & 3 & 4 \\
\hline 1) Social support & 2.82 & 0.68 & $\mathbf{0 . 7 3}$ & & & \\
2) Cultural capital & 3.45 & 0.96 & $0.18^{* *}$ & $\mathbf{0 . 8 0}$ & & \\
$\begin{array}{l}\text { 3) Relative deprivation } \\
\text { 4) Compensatory }\end{array}$ & 2.33 & 0.78 & $-0.26^{* *}$ & $-0.45^{* *}$ & $\mathbf{0 . 7 1}$ & \\
\begin{tabular}{l} 
consumption \\
\hline
\end{tabular} & 2.26 & 0.95 & $-0.31^{* *}$ & $-0.42^{* *}$ & $0.51^{* *}$ & $\mathbf{0 . 7 8}$ \\
\hline
\end{tabular}

a. $\mathrm{n}=357$; b. matrix diagonal represents AVE square root value; $\mathrm{c}$. ${ }^{\star}$ represents $\mathrm{p}<0.05 ;{ }^{\star *}$ represents $\mathrm{p}<$ 0.01 . 


\subsection{Descriptive Statistical Analysis}

Descriptive statistics of the variables included in this study are shown in Table 1. Results show that there is a statistically significant correlation between culture capital, relative deprivation, conspicuous consumption, and compulsive consumption. In addition, the correlation coefficients between the variables are less than 0.7 , that is, weak to moderate strengths of relationships, and the results of collinear diagnosis of the regression equation show that the variance expansion factor (VIF) of each variable is between 1.075 and 1.306, indicating that there is no serious multicollinearity between independent variables.

\subsection{Hypotheses Testing}

\subsubsection{Main Effect Test}

According to the results in Table 2, it can be seen from M3, M4, M5, and M6that cultural capital is statistically significant and negatively correlated with compensatory consumption $(\beta=-0.42, \beta=-0.42, \beta=-0.24, \beta=-0.23$; $\mathrm{p}<$ 0.01 ) without considering relative deprivation, assuming $\mathrm{H} 1$ is supported; $\mathrm{M} 1$ knows that culture Capital and relative deprivation have a significant negative impact $(\beta=-0.52, \mathrm{p}<0.01)$, assuming $\mathrm{H} 2$ is supported.

\subsubsection{Mediating Effect Test}

From the comparison of the models M3 and M5 in Table 2, when the cultural capital and the relative deprivation simultaneously enter the regression equation for compensatory consumption behavior, the negative impact of cultural capital on compensatory consumption is significantly reduced $(\beta=-0.24, \mathrm{p}<0.01)$, and the relative deprivation is significantly positively correlated with compensatory consumption $(\beta=0.46, \mathrm{p}<0.01)$, so the relative deprivation plays a partial mediating role between cultural capital and compensatory consumption, assuming $\mathrm{H} 3$ and H4 are supported.

Table 2. Results of hierarchical regression analysis.

\begin{tabular}{|c|c|c|c|c|c|c|}
\hline \multirow{2}{*}{ Variable } & \multicolumn{3}{|c|}{ Relative deprivation } & \multicolumn{3}{|c|}{ Compensatory consumption } \\
\hline & M1 & M2 & M3 & M4 & M5 & M6 \\
\hline Sex & $-0.12^{*}$ & -0.12 & $-0.32^{* *}$ & $-0.43^{* *}$ & $-0.45^{* *}$ & $-0.40^{* *}$ \\
\hline Age & 0.06 & 0.05 & 0.01 & 0.01 & -0.00 & -0.00 \\
\hline Job & -0.07 & -0.04 & -0.05 & -0.03 & -0.01 & -0.02 \\
\hline Educate & $-0.11^{*}$ & -0.10 & $-0.15^{* *}$ & $-0.14^{*}$ & $-0.13^{*}$ & $-0.11^{*}$ \\
\hline Income & 0.09 & 0.09 & $0.11^{*}$ & $0.11^{*}$ & 0.08 & $0.09^{*}$ \\
\hline Cultural capital & $-0.52^{* *}$ & $-0.50^{* *}$ & $-0.45^{* *}$ & $-0.42^{* *}$ & $-0.24^{* *}$ & $-0.23^{* *}$ \\
\hline Relative deprivation & & & & & $0.46^{* *}$ & $0.39^{* *}$ \\
\hline Social support & & $-0.19^{* *}$ & & $-0.22^{* *}$ & & $-0.15^{*}$ \\
\hline Cultural capital $\times$ Social support & & $0.18^{*}$ & & $0.30^{* *}$ & & $0.24^{* *}$ \\
\hline $\mathrm{R}^{2}$ & 0.23 & 0.27 & 0.29 & 0.35 & 0.38 & 0.41 \\
\hline$\Delta \mathrm{R}^{2}$ & $-0.12^{*}$ & 0.04 & & 0.06 & & 0.03 \\
\hline
\end{tabular}

a. $\mathrm{n}=357 ; \mathrm{b} .{ }^{*}$ represents $\mathrm{p}<0.05 ;{ }^{* *}$ represents $\mathrm{p}<0.01$. 


\subsubsection{Moderating Effect Test}

It can be seen from M2 in Table 2 that the interaction between cultural capital and social support significantly affects the relative deprivation $(\beta=0.18, \mathrm{p}<$ 0.05 ), indicating that social support plays a moderating role in the relationship between cultural capital and relative deprivation, assuming $\mathrm{H} 5$ is supported. The corresponding moderating effect diagram is shown in Figure 2.

\subsubsection{Mediated Moderating Effect Test}

It can be seen from M4 in Table 2 that the interaction term between cultural capital and social support significantly positively affects compensatory consumption $(\beta=0.30, \mathrm{p}<0.01)$ without considering the effect of relative deprivation. It is also known from M6 that when the relative deprivation is added to the regression of compensatory consumption, the influence of the interaction term on compensatory consumption is reduced $(\beta=0.24, \mathrm{p}<0.01)$, and the relative deprivation and compensatory consumption are still significant positive correlation $(\beta=0.39, \mathrm{p}<0.01)$, so the relative deprivation mediates the influence of the interaction between cultural capital and social support on compensatory consumption, assuming $\mathrm{H} 6$ is supported.

\section{Discussion and Conclusions}

\subsection{Research Conclusions}

From the perspective of relative deprivation theory, this study uses quantitative research methods to examine and verify the relationship between cultural capital and compensatory consumption propensity, and examines the role of relative deprivation and social support. Research indicates:

1) Cultural capital has a significant negative impact on compensatory consumption. In general, individuals with higher cultural capital tend to have higher levels of non-cognitive ability and rationality, and thus the possibility of compensatory consumption is relatively lower.

2) Cultural capital can affect compensatory consumption through relative deprivation. In the process of individual consumer socialization, cultural capital shapes the individual's self-concept and consumer taste. Therefore, in the social comparison with the reference group for consumption, individuals with high cultural capital tend to be more determined and confident, and experience less relative deprivation, and thus show a low level of compensatory consumer will.

3) Social support can moderate the relationship between cultural capital and compensatory consumption. This moderating effect can be played through relative deprivation. A high level of social support can alleviate the relative deprivation of low-cultural capital to individuals, help to repair individual negative emotions, and reduce compensatory consumption propensity.

\subsection{Marketing Enlightenment}

First, focus on the cultural attributes of compensatory products. Enterprises can implement targeted marketing programs based on the psychological needs 


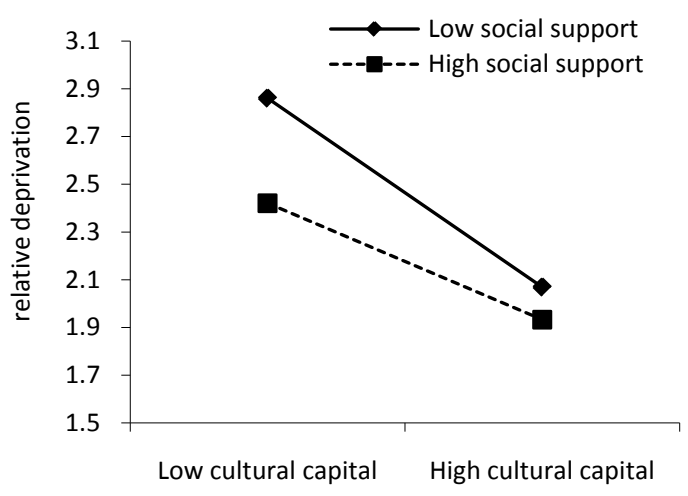

Figure 2. Moderating effect diagram.

of consumers at different levels of cultural capital. For individuals with high cultural capital, enterprises can convey the symbolic meanings of their products or services that are consistent with their self-concepts, such as status and taste, in the process of marketing promotion; for individuals with low cultural capital levels, the brand identity covered by the product and the brand association of the cultural elite should be strengthened.

Second, emphasize the social attributes of compensatory products. This study shows that in the process of consumer socialization, individuals are easily affected by the reference group, and they may choose to consume the same product in order to obtain the identity of the reference group. Therefore, in the process of marketing and promotion, enterprises need to pay attention to social attributes such as belonging and security brought by products to arouse consumers' emotional cognition and purchase intention.

Finally, as an irrational behavior, compensatory consumption is not conducive to the healthy development of consumers' mind and body, nor to the harmony and stability of society. Therefore, for customers who exhibit compensatory consumption characteristics, companies should appropriately guide and remind them to establish correct values and consumption habits. This kind of initiative to undertake social responsibility can not only help the company to win a good reputation, but also help the long-term stable cooperative relationship between the enterprise and the consumer.

\subsection{Insufficient Research and Prospects}

First, in terms of data, the cross-section data selected for this study may influence the determination of causality between variables. In addition, the sample range of the study is limited and lacks representativeness. Future research may consider more rigorous data collection and measurement methods. Secondly, although this paper explores the causes of compensatory consumption based on a new perspective of relative deprivation theory, it only studies the relative deprivation, but does not consider the impact of absolute deprivation. Based on this, future research can expand the mechanism of action and boundary conditions to further clarify the mechanism of deprivation and irrational consumption 
of vulnerable groups caused by the gap between rich and poor and class differentiation in the context of the rapid transformation of Chinese society.

\section{Conflicts of Interest}

The authors declare no conflicts of interest regarding the publication of this paper.

\section{References}

[1] Godart, F. and Yue, Z. (2014) Drivers of China's Desire for Luxury and Consequences for Luxury Brands. In: Atwal, G. and Bryson, D., Eds., Luxury Brands in Emerging Markets, Springer, Berlin, 119-129.

https://doi.org/10.1057/9781137330536_11

[2] Wang, Y. and Griskevicius, V. (2014) Conspicuous Consumption, Relationships, and Rivals: Women's Luxury Products as Signals to Other Women. Journal of Consumer Research, 40, 834-854. https://doi.org/10.1086/673256

[3] Veblen, T. (1994) The Theory of the Leisure Class. Courier Corporation, North Chelmsford.

[4] Rucker, D.D. and Galinsky, A.D. (2009) Conspicuous Consumption versus Utilitarian Ideals: How Different Levels of Power Shape Consumer Behavior. Journal of Experimental Social Psychology, 45, 549-555. https://doi.org/10.1016/j.jesp.2009.01.005

[5] Liu, W., Wang, H. and Chen, Z. (2014) Review and Prospect of Compensatory Consumption Research. Foreign Economics and Management, 36, 20-28.

[6] Rucker, D.D., Galinsky, A.D. and Dubois, D. (2012) Power and Consumer Behavior: How Power Shapes Who and What Consumers Value. Journal of Consumer Psychology, 22, 352-368. https://doi.org/10.1016/j.jcps.2011.06.001

[7] Shavitt, S., Jiang, D. and Cho, H. (2016) Stratification and Segmentation: Social Class in Consumer Behavior. Journal of Consumer Psychology, 26, 583-593.

https://doi.org/10.1016/j.jcps.2016.08.005

[8] Büchner, C., Cörvers, F., Traag, T., et al. (2012) How Do Education, Cognitive Skills, Cultural and Social Capital Account for Intergenerational Earnings Persistence? Evidence from the Netherlands. Social Science Electronic Publishing, Rochester. https://doi.org/10.2139/ssrn.2569028

[9] Bourdieu, P. (1984) Distinction: A Social Critique of the Judgment of Taste. Harvard University Press, Cambridge.

[10] Gromo, S. (1988) Compensatory Consumer: Elements of a Critical Sociology of Consumption. Proceedings of the 2 nd Conference on gender and Consumer Behavior, Salt Lake City, 27 April 1988, 65-85.

[11] Grunert, S. (1993) On Gender Differences in Eating Behavior as Compensatory Consumption. Proceedings of the 2 nd Conference on Gender and Consumer Behavior, Utah, 10 October 1993, 27-45.

[12] Ma, W. (2012) Relative Deprivation and Social Adaptation: Mediating and Regulating Effects. Acta Psychologica Sinica, 44, 377-387.

https://doi.org/10.3724/SP.J.1041.2012.00377

[13] Belk, R.W. (1988) Possessions and the Extended Self. Journal of Consumer Research, 15, 139-168. https://doi.org/10.1086/209154

[14] Song, X., Duan, C. and Wang, D. (2017) Research on the Influence of Pride on 
Consumer Status Consumption-Based on the Mediating Role of Cultural Capital and Economic Capital. Consumer Economy, No. 6, 48-55.

[15] Cui, N., Xu, W. and Liu, H. (2015) The Relationship between Cultural Capital and Brand Preference Intergenerational Generation. Economic Management, No. 2, 84-95.

[16] Abrams, D. and Grant, P.R. (2012) Testing the Social Identity Relative Deprivation (SIRD) Model of Social Change: The Political Rise of Scottish Nationalism. British Journal of Social Psychology, 51, 674-689. https://doi.org/10.1111/j.2044-8309.2011.02032.x

[17] Wilkinson, R.G. and Pickett, K.E. (2007) The Problems of Relative Deprivation: Why Some Societies Do Better than Others. Social Science \& Medicine, 65, 1965-1978. https://doi.org/10.1016/j.socscimed.2007.05.041

[18] Chipp, K., Kleyn, N. and Manzi, T. (2011) Catch Up and Keep Up: Relative Deprivation and Conspicuous Consumption in an Emerging Market. Journal of International Consumer Marketing, 23, 117-134. https://doi.org/10.1080/08961530.2011.543053

[19] Kim, H., Callan, M.J., Gheorghiu, A.I., et al. (2017) Social Comparison, Personal Relative Deprivation, and Materialism. British Journal of Social Psychology, 56, 373-392. https://doi.org/10.1111/bjso.12176

[20] Xiao, S. and Yang, D. (1987) The Impact of Social Support on Physical and Mental Health. Chinese Journal of Mental Health, No. 4, 183-187.

[21] Cohen, S., Underwood, L.G. and Gottlieb, B.H. (2000) Social Support Measurement and Intervention: A Guide for Health and Social Scientists. Psychiatric Services, 52, 1404-1404. https://doi.org/10.1176/appi.ps.52.10.1404

[22] Rosenbaum, M.S. (2008) Return on Community for Consumers and Service Establishments. Journal of Service Research, 11, 179-196. https://doi.org/10.1177/1094670508324298

[23] Dubois, D., Rucker, D.D. and Galinsky, A.D. (2012) Super Size Me: Product Size as a Signal of Status. Journal of Consumer Research, 38, 1047-1062. https://doi.org/10.1086/661890

[24] Hejase, A. and Hejase, H. (2013) Research Methods: A Practical Approach for Business Students. 2nd Edition, Masadir Inc., Philadelphia.

[25] Jin, X., Zhao, S., Cui, H., et al. (2017) The Influence of Status Perception Change on Consumer Status Consumption Behavior. Acta Psychologica Sinica, 49, 273-284. https://doi.org/10.3724/SP.J.1041.2017.00273 